\title{
Una mirada hacia la inseguridad alimentaria sudamericana
}

\author{
An overview of South American food insecurity
}

Edgar Manuel Aulestia-Guerrero (https://orcid.org/0000-0002-7381-1213) ${ }^{1}$

Edwin Daniel Capa-Mora (https://orcid.org/0000-0002-3479-2494) ${ }^{1}$

${ }^{1}$ Ciencias Biológicas, Biotecnología y Producción, Universidad Tecnica

Particular de Loja. San Cayetano Alto, Calle París. Loja Ecuador. emaulestia@hotmail.com

\begin{abstract}
The scope of this study is to examine the status of Food Insecurity (FI) in each country included in the South American community. To achieve this and considering that it is a current and multifactorial problem, its four pillars were analyzed: food availability, access, stability and use. As a result, it is observed that the misuse of food is the most likely cause of FI in the South American community, however, the lack of availability and inaccessibility of food causes great concern in certain states of the subregion. The most probable origin is the inadequate promotion of good eating habits, in addition to misguided policies that hinder the correct production and/or motivation of the peasants to continue carrying out their activities in agriculture. This problem needs to be addressed as soon as possible as, although it originates in not having the money and/or the necessary nutritional habits, it leads to serious health problems for the inhabitants. The consensus reached is that it is important to enhance policies to eradicate FI in South America, starting with support for food production, and following that up with nutritional interventions that improve the dietary habits of the inhabitants. Key words South America, Food security, Healthy eating, Dietary habits, Community intervention
\end{abstract}

Resumen El presente estudio pretende explorar el estado de Inseguridad Alimentaria (IA) en cada país perteneciente a la comunidad sudamericana. Para ello y considerando que es un problema vigente $y$ de carácter multifactorial, se analizaron sus cuatro pilares: disponibilidad, acceso, estabilidad y utilización de alimentos. Como resultado, se observa que la mala utilización de alimentos, es la causa más propensa a causar IA en la comunidad sudamericana, no obstante, la indisponibilidad e inaccesibilidad hacia los alimentos, causa gran preocupación en ciertos estados de la subregión. El origen más probable es la baja promoción de buenos hábitos alimenticios, además de políticas erradas que impiden la correcta producción $y / o$ motivación de los campesinos a seguir ejerciendo sus actividades en el agro. Este problema debe ser mitigado lo antes posible, ya que, aunque empieza por no tener el dinero $y / o$ los hábitos alimenticios necesarios, termina con graves problemas de salud hacia los pobladores. Consideramos importante fortalecer politicas para erradicar la IA sudamericana, iniciando con el apoyo hacia la producción de alimentos, para finalizar con intervenciones nutricionales que permitan mejorar los hábitos alimenticios de los habitantes.

Palabras clave América del Sur, Seguridad alimentaria, Alimentación saludable, Hábitos alimenticios, Intervención comunitaria 


\section{Introducción}

Para el año 2016, se estima que 815 millones de personas padecieron de subalimentación crónica, una disminución significativa comparada a los cerca de 900 millones que padecían de este mal en el año $2000^{1}$. Además, para el año 2011, se cree que se ha logrado la tasa más baja de mortalidad en niños por causas nutricionales, con 6,9 millones, comparado a los 12 millones que sucumbían 20 años atrás ${ }^{2}$. Así mismo, se estima que a nivel mundial, la prevalencia de la desnutrición infantil ha disminuido, del 29,5\% en el año 2005, a 22,9\% en el año $2016^{1}$, además de los niveles de pobreza, que para el año 1990 inquietaba a cerca de la mitad de la población mundial, para el 2015 afecta solamente a un $14 \%{ }^{3}$.

Sin embargo, en un comunicado realizado por la Organización de las Naciones Unidas para la Alimentación y la Agricultura (FAO) en el 2017, este asegura que los resultados hacia la erradicación de la inseguridad alimentaria se han visto truncados, sumándose esta vez a la problemática, altas incidencias de sobre peso y obesi$\operatorname{dad}^{1}$.

La inseguridad alimentaria es atribuida a diversos factores, como por ejemplo: inadecuadas políticas, toma decisiones y el bajo compromiso de autoridades estatales para afrontar el hambre y la pobreza de sus pueblos; así mismo, la falta de oportunidades laborales, la distribución desigual de los recursos estatales y la ausencia de políticas de apoyo hacia el sector agro productivo para asegurar la estabilidad alimentaria de la población; y las condiciones medioambientales extremas (inundaciones, sequías, etc.), sumada a la baja gestión gubernamental para enfrentar dicha problemática y la insuficiente preparación campesina que asegure la conservación de los recursos naturales, son factores que perjudican seriamente la estabilidad alimentaria global ${ }^{1,4}$.

A pesar de ello, en los próximos años, el descontrolado crecimiento poblacional será un reto para la sostenibilidad de la seguridad alimentaria, debido a que se deberá aumentar la producción de alimentos, luchar contra el cambio climático y conservar los recursos naturales de manera conjunta ${ }^{4}$. Por lo cual, es necesario explorar información y/o alternativas que permitan conocer las causas que propician esta problemática, con la intención de que un futuro cercano, se pueda hacer frente de mejor manera, a las consecuencias que acarrea la inseguridad alimentaria ${ }^{5}$.

Bajo esta perspectiva y considerando que la inseguridad alimentaria es un problema vigente y de carácter multifactorial, misma que causa gran preocupación a nivel mundial por su estrecha relación con altas tasas de mortalidad, enfermedades degenerativas y demás situaciones que ponen en riesgo la sobrevivencia y bienestar social de la población ${ }^{6}$, este trabajo pretende explorar el estado de la seguridad alimentaria en cada país perteneciente a la comunidad sudamericana.

\section{Metodología}

\section{Marco geográfico}

Sudamérica cuenta con un aproximado de 398'320.024 de habitantes y una extensión cercana a los $17^{\prime} 823.754 \mathrm{~km}^{2}$, donde se puede encontrar 12 países muy bien definidos: Argentina, Bolivia, Brasil, Chile, Colombia, Ecuador, Guyana, Paraguay, Perú, Surinam, Uruguay y Venezuela. Posee cerca de 27 tipos de climas según la clasificación de Köppen, mismos que son influenciados por su ubicación geográfica (tropical al centro y fría antártica al sur), sus océanos (variación de temperaturas marítimas y diversas corrientes) y la presencia de la cordillera de los andes (diversas escalas altitudinales), territorio enriquecido por la presencia de tres de los ríos más importantes a nivel mundial: el Amazonas, Orinoco y la Plata, y del acuífero Guaraní, uno de las más grandes en cuanto a recursos hídricos subterráneos, por lo cual, se le atribuye poseer el $28 \%$ del agua dulce global; en consecuencia, cerca del 22\% del territorio sudamericano está cubierto de selvas, permitiéndole además, desarrollar actividades agropecuarias de manera sustentable ${ }^{7}$ y poseer grandes reservas petroleras y mineras, con lo cual, la economía de la subregión, se enriquece de forma extraordinaria ${ }^{8}$.

\section{Recopilación de datos}

\section{Disponibilidad}

Se comparó la cantidad que produce cada país de estudio, de los principales alimentos recomendados por la FAO para poseer una buena nutrición y la cantidad de estos alimentos que necesita cada país, para alimentar adecuadamente a cada habitante.

Para la primera variable, se consideró los alimentos más comúnmente producidos a nivel mundial y que poseen gran importancia nutricional: energéticos (cereales), proteicos (leguminosas, carnes), frutas y verduras, mismos que fueron consultados por cada país en toneladas 
anuales. Esta información fue obtenida a través del portal de Datos de Alimentación y Agricultura de la Organización de las Naciones Unidas (FAOSTAT).

Para la segunda variable, se consideró lo recomendado por el Departamento de Agricultura de los Estados Unidos (USDA), donde menciona que, para un buen estado nutricional de una persona promedio que necesita 2.000 calorías diarias, es necesario consumir $0,17 \mathrm{~kg}$ de alimentos energéticos (cereales), 0,165 kg de alimentos proteicos (legumbres y carnes) y $0,4 \mathrm{~kg} g$ de frutas y verduras para el complemento de micronutrientes, vitaminas y minerales ${ }^{9}$, datos que fueron multiplicados por el número aproximado de habitantes de cada país y el número de días que tiene un año.

Finalmente, se utilizó la fórmula 1 para obtener el porcentaje de producción nacional que cada país deberá utilizar, para amortiguar las necesidades alimenticias y nutricionales de cada habitante.

$$
\mathrm{x}=\frac{\mathrm{n}^{\star} 100}{\mathrm{~d}}
$$

Donde:

$\mathrm{x}=$ Producción nacional requerida para alimentar a cada habitantes;

$\mathrm{n}=$ Cantidad de alimento necesaria para alimentar a cada habitantes;

$\mathrm{d}=$ Cantidad de alimento disponible en cada país;

100 = factor de conversión a porcentaje.

\section{Accesibilidad}

Por cada país, se consultó y comparó el suelo básico y el costo aproximado de la canasta básica de alimentos, con la intención de conocer el porcentaje de sueldo que debe ser utilizado con fines de alimentación, por parte de la comunidad sudamericana.

Los antecedentes de cada país de estudio, fueron colectados a través del portal en línea: FINANCIALRED, mismo que proporciona datos financieros de toda la región latinoamericana ${ }^{10}$. No obstante, dicha información fue verificada y modificada por los autores, con la intención de comprobar la autenticidad de la información, por lo cual, se consideró sumamente importante, visitar páginas oficiales de cada país estudiado.

Por último, se utilizó la fórmula 2 para calcular el porcentaje de sueldo básico mensual que debe ser utilizado para solventar el costo de la canasta básica de alimentos, en cada estado sudamericano.

$$
\mathrm{y}=\frac{\mathrm{CCBF}^{\star} 100}{\mathrm{SBM}}
$$

Donde:

$\mathrm{y}=$ Solvencia de la canasta básica de alimentos;

CCBF = Costo de la canasta básica familiar de alimento;

SBM = Sueldo básico mensual de cada país;

$100=$ factor de convertibilidad a porcentaje.

\section{Estabilidad}

La total permanencia en la producción de alimentos, es un factor fundamental hacia la certificación de la seguridad alimentaria, ya que, del sector agropecuario depende, en gran medida, poseer la cantidad, variedad e inocuidad de alimentos para satisfacer las necesidades nutricionales de los habitantes ${ }^{6}$.

Por tal motivo, consideramos sumamente relevante, estudiar las consecuencias que los desastres naturales pueden ocasionar hacia la sostenibilidad agro-productiva, ocasionando como efectos colaterales, serios problemas hacia la seguridad alimentara de la comunidad ${ }^{1}$.

La información fue obtenida, a través del último informe de la Oficina de las Naciones Unidas para la Reducción del Riesgo de Desastres, denominado "Enfoque para la gestión del riesgo de desastres en América del Sur", con lo cual, se pudo estudiar qué países sudamericanos son más propensos a verse perjudicados, además de analizarse las causas que los propician y las consecuencias que ocasionan hacia la estabilidad económica y alimentaria de la población sudamericana.

\section{Utilización de los alimentos}

Debido a que la inseguridad alimentaria por utilización de alimentos, puede abarcar diversos parámetros, cabe indicar que existen tres causas con mayor incidencia en la actualidad, siendo la subalimentación (dieta alimentaria insuficiente), obesidad (exceso de grasa acumulada en el cuerpo) y retraso en el crecimiento (aumento insuficiente de tamaño), aquellas con mayor preocupación a nivel mundial ${ }^{1}$.

Por tal motivo, se consideró relevante estudiar estos tres parámetros y determinar el porcentaje de habitantes aquejados por este mal en la comunidad sudamericana. Para lo cual, se recolectó la información a través del último informe sobre seguridad alimentaria, mismo que fue publicado por la FAO en el año 2017 y que cuenta con datos de toda la región latinoamericana, incluida la subregión de América del Sur. 


\section{Resultados}

\section{Disponibilidad de alimentos}

La Tabla 1, detallada a continuación, muestra la cantidad de alimento que produce cada país en la actualidad y la cantidad de alimento que demandan los mismos, para solventar las necesidades alimenticias y/o nutricionales de sus habitantes.

A continuación, se muestra la Tabla 2, misma que detalla el porcentaje de la producción de cada país, que deberá ser utilizado para suplir la necesidad alimenticia de cada habitante.

Los cálculos muestran que, a nivel subregional, se está utilizando cerca del 30,9\% de la producción total de alimentos energéticos y el $34,8 \%$ de alimentos proteicos para solventar las necesidades de la comunidad sudamericana, no obstante, se evidencia que existen un déficit aproximado del 5,9\% de producción de frutas y verduras.

Así mismo, se observa que Venezuela, cuenta con un déficit en la producción de alimentos energéticos, frutas y verduras; además, Surinam cuenta con insuficiente producción de alimentos proteicos, frutas y verduras; y Uruguay, Ecuador y Argentina cuentan con deficiente producción de frutas y verduras.

\section{Accesibilidad económica hacia los alimentos}

A continuación, la Tabla 3 muestra el porcentaje de salario que cada país sudamericano, debe- rá destinar a la adquisición de alimentos para el núcleo familiar.

Observamos que Venezuela, Perú, Paraguay, Guyana, Colombia, Surinam, Argentina y Ecuador, son los países más vulnerables para acceder a una canasta básica de alimentos. No obstante, la FAO menciona que, si una familia depende de más del 50\% de sus ingresos para su alimenta-

Tabla 2. Producción requerida para alimentar a cada habitante.

\begin{tabular}{lccc}
\hline & $\begin{array}{c}\text { Energéticos } \\
(\%)\end{array}$ & $\begin{array}{c}\text { Proteicos } \\
(\%)\end{array}$ & $\begin{array}{c}\text { Frutas y } \\
\text { verduras } \\
(\%)\end{array}$ \\
\hline Argentina & 4,26 & 3,52 & $-21,67$ \\
Bolivia & 38,34 & 9,55 & 72,30 \\
Brasil & 15,50 & 7,42 & 93,50 \\
Chile & 14,92 & 14,48 & 35,10 \\
Colombia & 80,06 & 38,14 & 69,44 \\
Ecuador & 37,36 & 56,48 & $-32,58$ \\
Guyana & 8,60 & 42,21 & 74,52 \\
Paraguay & 5,99 & 3,12 & 29,77 \\
Perú & 35,78 & 69,55 & 48,15 \\
Surinam & 12,71 & $-25,77$ & $-38,10$ \\
Uruguay & 6,44 & 3,68 & $-47,41$ \\
Venezuela & $-10,11$ & 35,03 & $-54,53$ \\
Total & 30,9 & 34,8 & 105,9 \\
\hline Nota: Cifras negativas, muestran el déficit de alimento de un \\
país.
\end{tabular}

Tabla 1. Disponibilidad y necesidad de alimento en los países de América del Sur

\begin{tabular}{lrrrrrr}
\hline & \multicolumn{2}{c}{ Disponibilidad de alimentos (tn) } & \multicolumn{2}{c}{ Necesidad de alimentos (tn) } \\
\hline \multicolumn{1}{c}{ País } & Energéticos & Proteicos & $\begin{array}{c}\text { Frutas y } \\
\text { verduras }\end{array}$ & Energéticos & Proteicos & \multicolumn{1}{c}{$\begin{array}{c}\text { Frutas y } \\
\text { verduras }\end{array}$} \\
\hline Argentina & 63.848 .731 & 75.063 .387 & 5.011 .716 & 2.719 .217 & 2.639 .240 & 6.398 .158 \\
Bolivia & 1.791 .112 & 6.980 .852 & 2.234 .536 & 686.645 & 666.450 & 1.615 .636 \\
Brasil & 82.858 .424 & 167.942 .719 & 32.325 .449 & 12.845 .095 & 12.467 .298 & 30.223 .752 \\
Chile & 7.605 .342 & 7.605 .342 & 7.605 .342 & 1.134 .646 & 1.101 .274 & 2.669 .756 \\
Colombia & 3.803 .109 & 7.747 .941 & 10.316 .066 & 3.044 .607 & 2.955 .060 & 7.163 .782 \\
Ecuador & 2.766 .657 & 1.776 .121 & 1.639 .480 & 1.033 .505 & 1.003 .108 & 2.431 .776 \\
Guyana & 538.450 & 106.446 & 146.153 & 46.289 & 44.928 & 108.916 \\
Paraguay & 7.154 .320 & 13.349 .127 & 3.386 .837 & 428.455 & 415.854 & 1.008 .130 \\
Perú & 5.490 .759 & 2.741 .611 & 9.599 .825 & 1.964 .503 & 1.906 .724 & 4.622 .360 \\
Surinam & 278.166 & 25.481 & 51.516 & 35.369 & 34.328 & 83.220 \\
Uruguay & 3.358 .168 & 5.699 .659 & 267.757 & 216.368 & 210.005 & 509.102 \\
Venezuela & 1.742 .152 & 5.370 .498 & 2.073 .549 & 1.938 .194 & 1.881 .188 & 4.560 .456 \\
Total & 181.235 .390 & 294.409 .184 & 74.658 .226 & 26.092 .894 & 25.325 .456 & 61.395 .044 \\
\hline
\end{tabular}

Fuente: Elaboración propia a partir de FAOSTAT (FAO en línea). 
Tabla 3. Salario mensual destinado a compra de alimentos.

\begin{tabular}{llccc}
\hline \multicolumn{1}{c}{ País } & \multicolumn{1}{c}{ Tipo de moneda } & $\begin{array}{c}\text { Salario básico } \\
\mathbf{2 0 1 8} \\
\text { (moneda local) }\end{array}$ & $\begin{array}{c}\text { Costo CBA 2018 } \\
\text { (moneda local) }\end{array}$ & $\begin{array}{c}\text { Dinero utilizado } \\
\text { (\%) }\end{array}$ \\
\hline Argentina & Pesos argentinos & 9.500 & 16.644 & $-42,9$ \\
Bolivia & Bolivianos & 2.000 & 1071 & 53,55 \\
Brasil & Reales & 965 & 400 & 41,45 \\
Chile & Pesos chilenos & 276.000 & 137.458 & 49,80 \\
Colombia & Pesos colombianos & 869.453 & 1.330 .000 & $-34,6$ \\
Ecuador & Dólares & 386 & 490 & $-21,2$ \\
Guayana & Dólar guyanés & 35.000 & 40.980 & $-14,6$ \\
Paraguay & Guaraníes & 2.200 .000 & 3.656 .266 & $-39,8$ \\
Perú & Soles & 850 & 1.515 & $-43,9$ \\
Surinam & Dólar Surinamés & 600 & 1.390 & $-56,8$ \\
Uruguay & Pesos uruguayos & 13.430 & 12.252 & 91,23 \\
Venezuela & Bolívares & 798.510 & 5.594 .119 & $-85,7$ \\
\hline
\end{tabular}

Fuente: FINANCIALRED (en línea), modificado por los autores. Nota: Cifras negativas, muestran el déficit monetario de un país.

ción, tiene más probabilidad de sumirse en problemas de inseguridad alimentaria, ya que deberán destinar parte de esos ingresos hacia otros gastos necesarios como: salud, educación, vestimenta, etc. ${ }^{1}$, por lo cual, Bolivia y Uruguay se suman a la lista de países que pueden ver perjudicada su seguridad alimentaria por falta de ingresos.

\section{Estabilidad de los alimentos}

Según la Oficina de las Naciones Unidas para la Reducción del Riesgo de Desastres (UNISDR), toda Sudamérica está expuesta a diversos tipos de catástrofes naturales. No obstante, se pudo identificar que, los desastres volcánicos, maremotos (sismos e inundaciones), aquellos que tienen que ver con variaciones hidrometeorológicas (sequías y/o lluvias), deforestaciones e incendios forestales, son los que han causado mayores daños a la comunidad sudamericana ${ }^{7}$.

Estos siniestros han atentado, a lo largo de la existencia de la subregión, a la estabilidad socio-económica de los sudamericanos, lo cual ha incidido en gran parte, para que la economía de los países subdesarrollados de América del Sur, puedan frenar sus avances hacia una economía diga que proporcione la estabilidad, tanto alimentaria como económica de estos pueblos, ya que sus consecuencias hacia la salud de los habitantes, como al campo agro-productivo, han sido devastadoras ${ }^{11,12}$.

\section{Utilización de los alimentos}

La Tabla 4, detallada a continuación, muestra las consecuencias que ha acarreado la mala utilización de alimentos, por parte de los habitantes sudamericanos.

Se puede observar que, a nivel regional, la prevalencia de subalimentación es del 8,4\%, siendo Bolivia el país con mayor incidencia, seguido por Venezuela, Ecuador y Paraguay quienes cuentan con prevalencias mayores al 10\%. Así mismo, se puede evidenciar que el porcentaje de habitantes con problemas de obesidad, es muy notorio en todos los países de estudio.

Además, se puede percibir que el retraso en el crecimiento en niños acarrea cifras altas, siendo Chile, aquel país con niveles más bajos de este problema (1,5\%). No obstante, países como Bolivia y Ecuador superan el 20\% de incidencia, Perú, Venezuela, Colombia, Guyana, Uruguay y Paraguay sobrepasan el 10\% y Argentina, Surinam y Brasil poseen cifras menores al $10 \%$, sin embargo, aún son estadísticas preocupantes.

\section{Discusión}

\section{Disponibilidad}

La disponibilidad de alimentos en Venezuela, se vio perjudicada desde que el país decidió aban- 


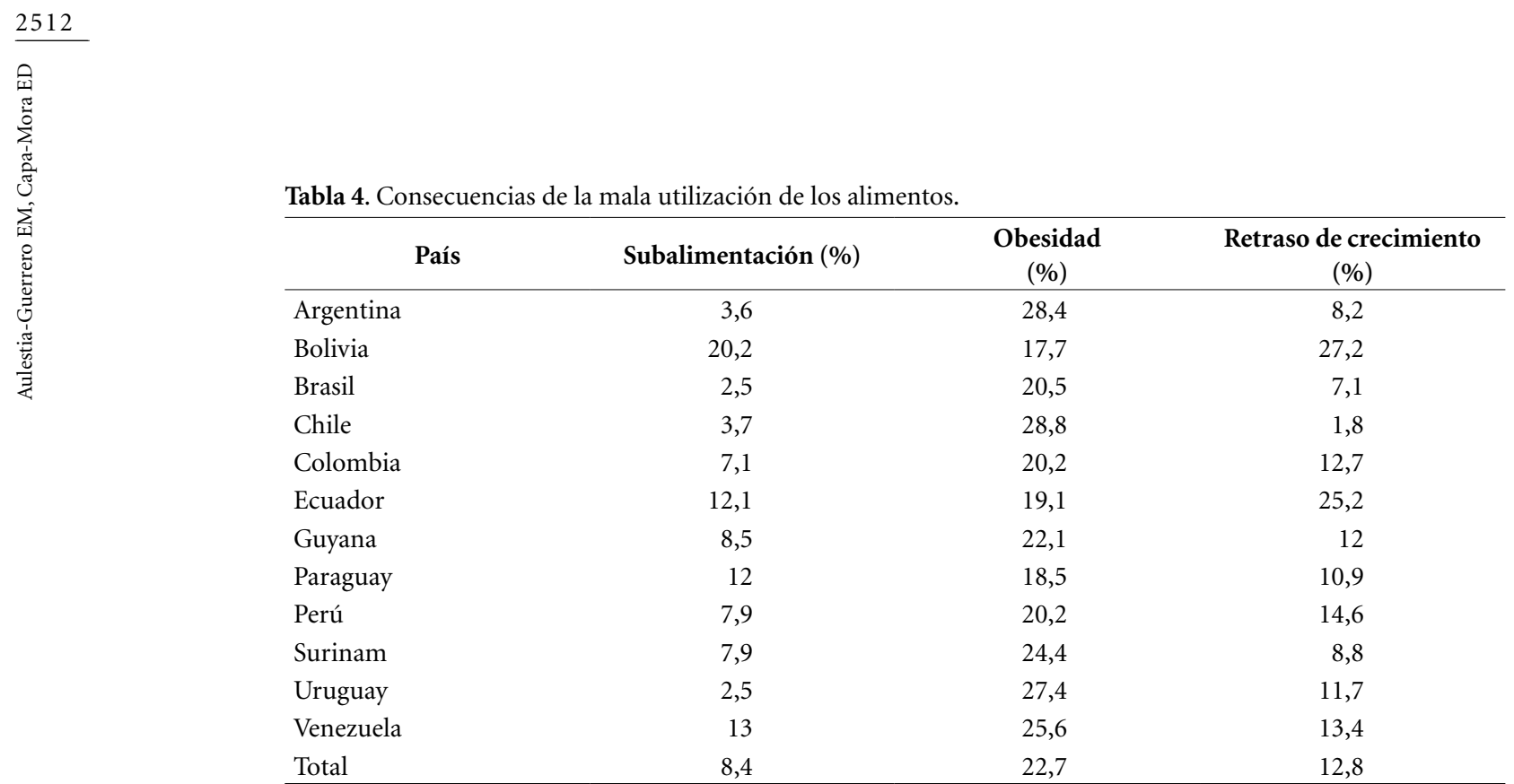

Fuente: FAO \& OPS, 2017; FAOSTAT (FAO en línea).

donar el sector agropecuario, para volcarse hacia una economía netamente petrolera, resultando en una sobre-población del sector urbano y ocasionando que apenas el 12,3\% de venezolanos se dedique a actividades del agro, lo cual, explica la escases de varios productos alimenticios ${ }^{13}$.

Para Surinam, aunque no existe información oficial, la ONU menciona que el problema puede ser, debido al bajo apoyo gubernamental hacia el fortalecimiento del sector agropecuario nacional, y así, producir la cantidad suficiente de alimentos para brindar a sus gobernados ${ }^{14}$.

En el caso de Argentina y Uruguay, la insuficiencia de frutas y verduras, se debe la escasa promoción de las cualidades nutritivas de estos alimentos hacia los pobladores, lo que ocasiona, como resultado, que no haya una producción suficiente debido a que la demanda de la misma, no resulta rentable para expandir su producción ${ }^{15,16}$.

Por último, en el caso de Ecuador, el estado ha dedicado gran inversión hacia el sector agropecuario, no obstante, se conoce que toda la producción fue dedicada a monocultivos comerciales y muchas veces, los acontecimientos hidrometereológicos perjudican el agro ecuatoriano, contribuyendo a la pérdida de las cosechas, y ocasionando indisponibilidad de estos alimentos ${ }^{17}$.

Por todo esto, se cree que la inversión gubernamental en el sector agropecuario debe ser un pilar fundamental para mejorar la economía de los habitantes pertenecientes a países en desarrollo, ya que, gran parte de los pobladores rurales dependen, de alguna u otra forma, de la pequeña agricultura ${ }^{18}$. Así mismo, este sector es de gran relevancia para certificar una seguridad alimentaria digna, debido a la variedad e inocuidad de alimentos que puede brindar este sector si es producido de manera correcta, por lo cual, es recomendable programas interventores que proporcionen la información adecuada de producción hacia los pequeños y medianos productores, con la finalidad de fortalecer el campo agropecuario, y con ello, la economía y alimentación de los habitantes ${ }^{19}$.

\section{Acceso}

Para Venezuela, Argentina, Bolivia, Ecuador y Uruguay, el problema radica en la gran inflación alimentaria que han tenido que atravesar dichos países, lo que ha perjudicado la estabilidad de precios de los alimentos básicos y haciendo muy complicada la adquisición de los mismos ${ }^{20-22}$, problema que se da por malas prácticas económicas estatales, sobre demanda y/o déficit de algún producto alimenticio ${ }^{23}$.

Así mismo, en el caso de Perú, Paraguay y Bolivia, analistas aseguran que el problema se da, debido a la falta de políticas gubernamentales que permitan la producción de alimentos de manera adecuada por parte del sector agropecuario, ocasionando altos precios en los mismos, y como consecuencia, deberán destinar mayores sueldos para la compra de la canasta básica de alimentos básicos $^{24-26}$.

Por último, para el caso de Surinam y Guyana, el problema se debe a la extrema margina- 
ción social que soportan sus habitantes, ya que no existen políticas que aseguren la constante producción de alimentos, conllevando al sobre precio de los mismos, y como resultado, los habitantes tendrán mayores problemas para adquirirlos $^{14}$

Por todo esto, el desapego social y/o económico por parte de los gobiernos hacia sus pueblos, ha sido un factor que la FAO a tratado de erradicar, ya que, dicha problemática, tiene gran ligadura con la alta incidencia de inseguridad alimentaria ${ }^{27}$. Sin embargo, este objetivo no ha sido cumplido a cabalidad, ya que la pobreza y malnutrición en la población latinoamericana y mundial, se debe, muchas veces, a la mala gobernanza, debido a que los habitantes, mayormente los de sectores rurales, poseen poca atención por parte de los gobiernos para ser incluidos en programas que aseguren una economía digna ${ }^{28-30}$.

En este mismo sentido, se ha demostrado que la lucha contra esta problemática, se inicia cambiando la mentalidad de los gobernadores, ya que muchas veces, se tienen los recursos necesarios para acceder a los sectores vulnerables y combatir sus falencias, sin embargo, en muchas ocasiones, lo único que pretenden es especular, realizando proyectos poco significativos que, al final de la intervención, no han aportado convincentemente hacia los habitantes ${ }^{31,32}$, por lo cual, resulta importante crear estrategias que impidan que la política se convierta en una empresa lucrativa y sea utilizada para servir a los ciudadanos, con la intención de evitar que actores políticos, entidades gubernamentales y servidores públicos, se lucren de su puesto de trabajo ${ }^{33}$.

\section{Estabilidad}

Los eventos volcánicos a los que está expuesto Sudamérica, se deben a su ubicación sobre la subducción de la placa sudamericana con la placa nazca, convirtiéndola en una de las subregiones volcánicamente más activas a nivel mundial, especialmente en países como: Argentina, Bolivia, Chile, Colombia, Ecuador y Perú, ocasionando perjuicios hacia la seguridad alimentaria, debido a que las cenizas expulsadas desde los volcanes, pueden dañar cultivos y ganado, ocasionando pérdidas económicas y un déficit de alimentos, además de perjudicar la salud de los habitantes, ocasionando problemas para trabajar, obtener e ingerir alimentos adecuadamente ${ }^{34}$.

Los maremotos se deben a la presencia del cinturón de fuego del pacífico, considerado uno de los más agresivos a nivel mundial y que puede perjudicar a los países andinos de la subregión, además, la ubicación geográfica de la mayor parte de países cerca del perfil costanero, extiende la posibilidad de perjudicar la estabilidad alimentaria de la subregión, ya que las inundaciones ocasionan graves pérdidas agro-productivas, aumentando los índices de pobreza y ocasionando déficit de los alimentos básicos ${ }^{35}$.

Los desastres de origen hidrometereológico, sumada a la gran incidencia del fenómeno del niño y la niña, son problemas eminentes en la subregión, ocasionando que cada país deba estar preparado para combatir, tanto sequías como precipitaciones severas, con la finalidad de asegurar la sostenibilidad productiva interna y asegurar la alimentación de sus habitantes, dada la dificultad que resulta predecir la agresividad de dichos eventos climáticos ${ }^{36}$.

Por último, las acciones antropogénicas, como deforestación e incendios forestales a las que está expuesta la subregión, se deben a que gran parte de Sudamérica está provista de bosques, tanto siempre verdes como secos. Las causas que imperan sobre la deforestación, se deben, mayormente, a la venta de madera ${ }^{37}$, y los incendios forestales se atribuyen a la quema de bosques, muchas veces, para destinar a la agricultura $^{38}$. Ambos problemas son preocupantes, debido a que, luego de ser deforestados o incendiados, las lluvias pueden ocasionar erosiones, mayormente en laderas, ocasionando perjuicios a suelos que pudieron ser utilizados con fines netamente productivos ${ }^{39}$.

Por todo esto, resulta importante que cada estado cuente con la planificación adecuada para afrontar dichos eventos, considerando que los países con mayor inversión económica hacia la lucha contra desastres naturales, pueden hacerle frente de mejor manera y tienen mayor probabilidad de brindar seguridad social, económica y alimentaria a sus habitantes ${ }^{40}$. Además, las naciones deben adoptar políticas más drásticas con el tema de sobrepoblación, ya que ocasiona sobre urbanización y el deterioro de zonas, que muchas veces, no son aptas para ser habitables ${ }^{11}$, lo que incide para que el cambio climático y sus efectos sean más drásticos y perjudiquen con mayor frecuencia los sectores más vulnerables de la población ${ }^{41}$.

\section{Utilización}

Se ha demostrado que la población occidental tiene gran orientación hacia productos calóricos y grasos ${ }^{42}$, ocasionando enfermedades terapéu- 
ticas, cardiovasculares, dermatológicas, cánceres y dicho de manera general, menor esperanza de vida ${ }^{43,44}$. Además, se conoce que la cultura y educación alimenticia, incide notablemente en la seguridad alimentaria, ya que se tiene conceptos erróneos de la compra, consumo, correcta preparación y la importancia de variar los alimentos, conllevando una vida alimenticia monótona y repleta de alimentos que, aunque cumplen su función de satisfacer el hambre, no poseen los nutrientes necesarios para asegurar una correcta nutrición ${ }^{45}$.

Así mismo, se cree que los problemas de malnutrición en la actualidad, mayormente de sobre peso y obesidad, se debe al sedentarismo y poca actividad física de la población moderna, muchas veces, debido a que dedican mucho tiempo a trabajos de oficina ${ }^{46}$, por lo cual, resulta importante la promoción de buenos hábitos alimenticios y mayor actividad física, ya que se ha demostrado que estos dos factores, pueden incidir en la reducción de enfermedades degenerativas y demás problemas asociados a la mala utilización de los alimentos $^{47,48}$.

Es importante resaltar, que el impulso de estas actividades son más provechosas en la niñez, ya que es ahí, donde se puede formar personas conscientes y seguras de lo que ingieren ${ }^{42}$, por lo cual, las intervenciones deben enfocarse en todo el núcleo familiar, mucho más, a las madres y padres de familia, quienes deben ser los encargados de replicar estas costumbres a sus niños y adolescentes ${ }^{49}$.

Es así el caso de todos los país países de estudio, donde para Argentina, Bolivia, Brasil, Chile y Perú, investigadores aseguran que el alto consumo de hidratos de carbono y carnes, además de los malos balances alimentarios, están condenando la calidad de vida de los habitantes, ya que en muchos de los casos, las intervenciones realizadas para la mitigación del problema, han carecido de la fortaleza necesaria para combatir de manera adecuada los malos hábitos alimenticios de la población ${ }^{26,42,50-54}$.

Para el caso de Colombia, Ecuador, Paraguay y Uruguay, el problema se evidencia mayormente en sectores rurales, donde es muy complicado acceder a los suministros de manera igualitaria, ya que en muchos de los casos, no todos los alimentos necesarios llegan a estas comunidades, a esto se suma la dificultad de poder realizar intervenciones integrales y constantes en dichos sectores por parte de los organismos encargados, que podrían, de alguna manera, asegurar la calidad de vida de los colombianos ${ }^{5,17,55-59}$.
Por último, para Surinam y Guyana, aunque no existe mucha información sobre la problemática alimentaria, la ONU asegura que la marginación gubernamental hacia sus habitantes, ha incidido notablemente para que las personas obtengan altos índices de inseguridad alimentaria asociada a la utilización de los alimentos, ya que no existe, por lo menos, una planificación que demuestre el interés por parte de entidades gubernamentales, hacia la erradicación de los malos hábitos alimenticios y sus consecuencias, además de centros de salud y saneamiento que certifiquen la calidad de vida de los habitantes ${ }^{14}$. Mismo problema que aqueja a los venezolanos, quienes ven comprometida su seguridad alimentaria por utilización de los alimentos, debido a la falta de mecanismos gubernamentales, que contribuyan hacia la utilización segura de los alimentos por parte de la población, además de la falta de recursos económicos, lo que condiciona, en gran medida, la diversidad y calidad alimentaria de la población venezolana ${ }^{60}$.

\section{Conclusión}

Indiscutiblemente, Sudamérica es un sector vulnerable a padecer inseguridad alimentaria por estabilidad en la producción de alimentos, ya que su territorio está a expensas de sufrir diversos tipos de desastres naturales sin previo aviso. No obstante, este punto no es el más preocupante, ya que, al ser acontecimientos poco controlables, lo único que queda por hacer, es que cada país sudamericano, y sus habitantes, estén debidamente informados y capacitados para sobrellevar estos altercados.

Lo preocupante del caso, es que la disponibilidad de alimentos, su acceso y su utilización, están provocando índices mucho mayores de inseguridad alimentaria en la subregión sudamericana, ya que como se ha visto, algunos países cuentan con insuficiente producción interna de alimentos para brindar a sus gobernados, no cuentan con el dinero suficiente para acceder a una canasta básica de alimentos que asegure una alimentación variada e inocua, y poseen enfermedades degenerativas (que se evidencia mayormente en los niños) que comprometen de gran manera la calidad de vida de los pobladores.

Se cree que la sobre dependencia económica de una comunidad sobre sectores externos al agropecuario y erradas políticas que desmotivan a los productores a seguir ejerciendo sus actividades en el agro, han llevado a sufrir inseguridad 
alimentaria de algunas comunidades sudamericanas por indisponibilidad de alimentos y el inacceso a los mismos.

Además, consideramos que las pobres intervenciones gubernamentales $y / 0$ sus metodologías para realizar intervenciones comunitarias y tratar de erradicar los malos hábitos alimenticios presentes en la población, informando la importancia de variar los alimentos, cómo variarlos y/o consumirlos para ser aprovechados por el organismo de manera correcta, es la causa de inseguridad alimentaria por mala utilización de los alimentos.

Basados en las manifestaciones encontradas en la presente exploración, creemos que se debe intensificar y dar a notar mayor preocupación por parte de las autoridades, tanto gubernamentales y no gubernamentales, con la intención de fortalecer políticas que luchen contra la inseguridad alimentaria existente en la población sudamericana, considerando que, una población malnutrida, tendrá efectos negativos en el desarrollo económico y productivo de las naciones, debido a la gran inversión que las consecuencias de la malnutrición acarrean, además de que las personas, cuando padecen de dichas enfermedades, tendrán mayores problemas para poder estudiar y trabajar, ocasionando mayores perjuicios a la economía familiar y su seguridad alimentaria.

\section{Colaboradores}

EM Aulestia-Guerrero: Búsqueda de información; Revisión bibliográfica; Análisis de datos; Redacción y discusión. ED Capa-Mora: Búsqueda de información; Revisión bibliográfica; Análisis de datos; Redacción y discusión; Dirección de la investigación. 


\section{Referencias}

1. Organización de las Naciones Unidas para la Alimentación y la Agricultura (FAO), Organización Panamericana de la Salud (OPS). Panorama de la seguridad alimentaria y nutricional en América Latina y el Caribe. Santiago de Chile: FAO; 2017.

2. Fondo de las Naciones Unidas para la Infancia (UNICEF). Alianzas para la Nutrición Infantil. Alianzas para la Nutrición Infantil. Quito: UNICEF; 2014.

3. Organización de las Naciones Unidas (ONU). Objetivos de Desarrollo del Milenio: Informe 2015. Nueva York: ONU; 2015.

4. Comité de Seguridad Alimentaria Mundial (CSA). Marco estratégico mundial para la seguridad alimentaria y la nutrición (MEM) [Internet]. Vol. Cuarta ver. 2013. [cited 2018 Mar 15]. Available from: http:// www.fao.org/fileadmin/templates/cfs/Docs1213/gsf/ GSF_Version_2_SP.pdf

5. Lissbrant S. Seguridad alimentaria y nutricional en la región caribe: consecuencias de la desnutrición y buenas prácticas como soluciones. Investig Desarro 2015; 23(1):117-138.

6. Abdullah, Zhou D, Shah T, Ali S, Ahmad W, Ud Din I, Ilyas A. Factors affecting household food security in rural northern hinterland of Pakistan. J Saudi Soc Agric Sci 2017; 1-10.

7. Oficina de las Naciones Unidas para la Reducción del Riesgo de Desastres (UNISDR). América del Sur: Enfoque para la gestión del riesgo de desastres. Ginebra: UNISDR; 2015.

8. Cadena Montenegro JL. La geopolítica en Sudamérica: Conflictos potenciales y efectos globales. Investig Geogr 2011; 55:113-133.

9. Eich Drummond K, Brefere LM. Nutrition for foodservice and culinary professionals. Hoboken: John Wiley \& Sons; 2014.

10. Red de Blogs Especializados en Economia, Bolsa y Finanzas (FINANCIALRED). ¿Cuál es el salario minimo de los países de América Latina en 2018? [Internet]. 2018. [cited 2018 Abr 8]. Available from: http:// salariominimo.com. $\mathrm{mx} /$ comparativa-salario-minimo-latinoamerica/\#Cual_es_el_salario_minimo_de_ los_paises_de_America_Latina_en_2018

11. Capacci A, Mangano S. Las catástrofes naturales. Cuad Geogr Rev Colomb Geogr 2015; 24(2):35-51.

12. López Feldman AJ, Hernández Cortés D. Cambio climático y agricultura: una revisión de la literatura con énfasis en América Latina. Trimest Econ 2016; LXXXIII(4):459-496.

13. Domínguez F. Las complejidades de la seguridad y soberanía alimentaria en Venezuela. Rev Políticas Públicas 2016; (n. esp.):157-167.

14. Organización de las Naciones Unidas (ONU). Documento del programa para el país: Guyana y Suriname. New York: ONU; 2016.

15. Giai M, Veronesi G. Cadenas productivas y disponibilidad de alimentos en Argentina. Diaeta 2010; 28(131):27-31.

16. Olavarría S, Zacarías I. Obstaculizadores y facilitadores para aumentar el consumo de frutas y verduras en seis países de Latinoamérica. Arch Latinoam Nutr 2011; 61(2):154-162.
17. Salazar Marroquín S. Programas sociales de alimentación y nutrición del Estado ecuatoriano, estrategia nacional en favor de la alimentación saludable. Economía 2016; XLI(41):76-96.

18. Brown WJ. El papel de la agricultura en la reducción de la pobreza. Rev Mex Agronegocios 2013; 32:166-178.

19. Rodríguez-González S, Schneider S, Coelho-de-Souza G. Reconexión producción-consumo: cambio para la seguridad alimentaria y nutricional y el desarrollo rural. Agron Mesoam 2015; 26(2):373-385.

20. Organización de las Naciones Unidas para la Alimentación y la Agricultura (FAO). Precios de los alimentos en América Latina y el Caribe. Roma: FAO; 2015.

21. Sánchez Urribarrí RA. Venezuela (2015): Un régimen hídrido en crisis. Rev Cienc Política 2016; 36(1):365382.

22. Vallejo Zamudio LE. La inflación en Colombia. Apunt del Cenes 2016; 35(62):9-10.

23. Moreno-Brid JC, Rivas JC, Villarreal FG. Inflación y crecimiento económico. Investig Económica 2014; LXXIII(290):3-23.

24. Landini F. Problemas en la extensión rural paraguaya : modelos de extensión en la encrucijada. Cuad. Desarro. Rural 2012; 9(69):127-149.

25. Oxford Committee for Famine Relief (OXFAM). Pobreza y Desigualdad en el Perú: cuando el crecimiento económico no basta. Lima: OXFAM; 2014.

26. Delgado A, Naranjo Mejía H. Análisis de la vulnerabilidad de los hogares bolivianos a la inseguridad alimentaria en 2015. Investig Desarro 2017; 2(17):49-62.

27. Comisión Económica para América Latina y el Caribe (CEPAL). Agenda 2030 y los Objetivos de Desarrollo Sostenible: una oportunidad para América Latina y el Caribe. Santiago: CEPAL; 2016.

28. Ribotta S. Pobreza, hambre y justicia en América Latina y el Caribe. Debatiendo sobre la jsticia mientras 53 millones de Latinoamericanos sufren de hambre. Rev Electrónica Iberoam 2010; 4(1):144-180.

29. Sperandio N, Priore SE. Prevalence of household food insecurity and associated factors among Bolsa Família Program families with preschool children in Viçosa, Minas Gerais State, Brazil. Epidemiol e Serviços Saúde 2015; 24(4):739-748.

30. Nesengani TJ, Mudau MJ, Netshandama VO. Verification of the existence of food security projects in Limpopo Province, South Africa. South African J Agric Ext 2016; 44(2):52-58.

31. López Arévalo J, Núñez Medina G. Democratización de la pobreza en Chiapas. Econ Inf 2015; 393:62-81.

32. Boltvinik J, Damián A. Pobreza creciente y estructuras sociales cada vez más desiguales en México. Una visión integrada y crítica. Acta Sociológica 2016; 5(70):271-296.

33. Reveles Vázquez F. Problemas de la representación política y de la participación directa en la democracia. Estud Políticos 2017; 42:11-35.

34. Aguilar I, Alvarado GE. Pérdidas humanas y económicas causadas por el vulcanismo en Costa Rica entre 1953 y 2005. Rev Geológica en América Cent 2014; 51:93-128. 
35. Perozo Ynestroza MA, Marrero de León NV. Estimación del riesgo de inundación en urbanismos y zonas agrícolas ubicadas en la planicie del Río Coro, Estado Falcón, Venezuela. Suelo y Agua 2011; 20(3):5-11.

36. Ruíz Cabarcas AC, Pabón Caicedo JD. Efecto de los fenómenos de El Niño y La Niña en la precipitación y su impacto en la producción agrícola del departamento del Atlántico (Colombia). Cuad Geogr 2013; 22(2):35-54.

37. Armenteras D, Rodríguez Eraso N. Dinámicas y causas de deforestación en bosques de Latino América: Una revisión desde 1990. Colomb For 2014; 17(2):233246.

38. Huerta-Martínez FM, Ibarra-Montoya JL. Incendios en el bosque la primavera (Jalisco, México): un acercamiento a sus posibles causas y consecuencias. Ciencia UAT 2014; 9(1):23-32.

39. Hidalgo Montoya CA, Vega Gutiérrez JA. Estimación de la amenaza por deslizamiento detonados por sismos y lluvia (valle de Aburrá-Colmbia). Rev EIA 2014; 11(22):103-117.

40. Corominas O, Martí J. Estudio comparativo de los planes de actuación frente al riesgo volcánico (Chile, Costa Rica, El Salvador, Ecuador, España, México y Nicaragua). Rev Geológica en América Cent 2015; 52:33-56.

41. Díaz Cordero G. El cambio climático. Cienc Soc 2012; XXXVII(2):227-240.

42. Rodríguez RF, Palma LX, Romo BA, Escobar BD, Aragú GB, Espinoza OL, Norman LM, Gálvez CJ. Hábitos alimentarios, actividad física y nivel socioeconómico en estudiantes universitarios de Chile. Nutr Hosp 2013; 28(2):447-455.

43. Yeste D, Carrascosa A. El manejo de la obesidad en la infancia y adolecencia: de la dieta a la cirugía. Endocrinol $y$ Nutr 2012; 59(7):2012.

44. Finer N. Medical consequences of obesity. Med (United Kingdom) 2014; 43(2):88-93.

45. Briones Alonso E, Cockx L, Swinnen J. Culture and food security. Glob Food Sec 2018; 1-15.

46. Villagra C, Lomaglio D, Dip N. Hábitos de alimentación y actividad física en Antofagasta de la Sierra (3223 msnm), Catamarca, Noreste Argentino. Cuad la Fac Humanidades y Ciencias Soc Nac Jujuy 2014; (46):105-117.

47. Ávila García M, Huertas Delgado FJ, Tercedor Sánchez P. Programas de intervención para la promoción de hábitos alimentarios y actividad física en escolares españoles de Educación Primaria: revisión sistemática. Nutr Hosp 2016; 33(6):1438-1443.

48. Rocha Silva D, Martín-Mantillas M, Carbonell-Baeza A, Aparicio V, Delgado-Fernández M. Efectos de los programas de intervención enfocados al tratamiento del sobrepeso/obesidad infantil y adolescente. Rev Andal Med Deport 2014; 7(1):33-43.

49. Zambrano R, Colina J, Valero Y, Herrera H, Valero J. Evaluación de hábitos alimentarios y estado nutricional en adolescentes de Caracas, Venezuela. Nutr y Salur Pública 2013; 26(2):86-94.
50. Calvo EB, Aguirre P. Crisis de la seguridad alimentaria en la Argentina y estado nutricional en una población vulnerable. Arch Argent Pediatr 2005; 103(1):77-90.

51. Abeyá Gilardon EO. Una evaluación crítica de los programas alimentarios en Argentina. Salud Colect 2016; 12(4):589-604.

52. Mamani-Ortiz Y, Rojas-Salazar EG, Caero-Suarez RI, Choque-Ontiveros M del C. Prevalencia de desnutrición en niños y niñas en edad escolar del municipio de Vinto. Rev Médico-Científica "Luz y Vida" 2013; 4(1):36-40.

53. Souza NP, Lira PIC, Fontbonne A, Pinto FCL, Cesse EAP. La (des) nutrición y el nuevo patrón epidemiológico en un contexto de desarrollo y desigualdades. Cien Saude Colet 2017; 22(7):2257-2266.

54. Sánchez-Abanto J. Evolución de la desnutrición crónica en menores de cinco años en el Perú. Rev Peru Med Exp Salud Pública 2012; 29(3):402-405.

55. Hurtado Quintero C, Mejía C, Arango C, Chavarriaga LM, Grisales Romero H. Malnutrición por exceso y déficit en niños, niñas y adolescentes, Antioquia, 2015. Rev Fac Nac Salud Pública 2017; 35(1):58-70.

56. Calero C. Seguridad alimentaria en ecuador desde un enfoque de acceso a alimentos. Quito: Abya-Yala; 2011.

57. Echagüe G, Sosa L, Díaz V, Funes P, Rivas L, Granado D, Ruiz I, Zenteno J, Pistilli N. Malnutrition in indigenous and non-indigenous children under five years of age in rural areas, Paraguay. Memorias del Inst Investig en Ciencias la Salud 2016; 14(2):25-34.

58. Franca Tarragó O, Crestanello F, Müller A, Silveri A, Pons JE. El problema del sobrepeso y la obesidad en la niñez y adolescencia. Una mirada desde la Bioética. An la Fac Med 2017; 4(1):55-95.

59. Morinigo Isla G, Sánchez Bernal S, Sispanov Pankow V, Rolón Villalba G, Bonzi Arévalos C, Medina H, Cardozo K, Congo AR, Rivarola MB, Arbo LM. Perfil nutricional por antropometría de niños/as menores de 5 años del sistema público de salud, 2013. Pediatr (Asunción) 2015; 42(3):187-191.

60. López de Blanco M, Landaeta-Jiménez M, Herrera Cuenca M, Sifontes Y. La doble carga de desnutrición y obesidad en Venezuela. An Venez Nutr 2014; 27(1):77-87.

Artículo presentado en 23/06/2018

Aprobado en 03/12/2018

Versión final presentada en 05/12/2018 
